\title{
Siberian Snakes and Spin-Flipping in Storage Rings
}

\author{
B.B. Blinov \\ Randall Laboratory of Physics, University of Michigan, \\ Ann Arbor, Michigan 48109-1120
}

\begin{abstract}
A Siberian snake, which is a $180^{\circ}$ spin rotator, can be used to preserve electrons', protons' or light ions' beam polarization during acceleration by forcing the spin tune to be constant and equal to $1 / 2$, independent of the beam energy. A compact helical dipole snake, which creates only a small orbit excursion inside the snake, could be used in EPIC. Frequent polarization reversals, or spin-flips, of a stored polarized high-energy beam could greatly reduce the systematic errors of spin asymmetry measurements in a scattering asymmetry experiment. Such polarization reversals can be done by ramping an rf-dipole or an rf-solenoid magnet's frequency through an rf-induced depolarizing resonance. The strength of an $\mathrm{rf}$-solenoid decreases with the beam's energy due to the Lorenz contraction of the solenoid's longitudinal $\int B \cdot d l$, while the strength of an rf-dipole remains almost unchanged. Thus, it is more practical to use an rf-dipole spin-flipper in EPIC's electron and ion rings.
\end{abstract}

\section{INTRODUCTION}

In a circular accelerator ring, each proton's spin precesses around the vertical magnetic fields of the ring's bending dipoles, with a frequency, called the spin precession frequency $f_{s}$, that is related to the proton's circulation frequency $f_{c}$ by:

$$
f_{s}=f_{c} \nu_{s}
$$

where $\nu_{s}$ is the spin tune, which is the number of spin precessions during each turn around the ring.

If there are only vertical magnetic fields, then the vertical beam polarization remains unchanged; however, whenever there is a periodic horizontal magnetic field whose tune is equal to the spin tune, a depolarizing resonance occurs, which can destroy the polarization. The $\nu_{s}$ is proportional to the proton's energy via:

$$
\nu_{s}=G \gamma,
$$

where $\mathrm{G}=1.792847$ is the proton's anomalous magnetic moment, and $\gamma$ is the Lorentz energy factor; thus the protons will encounter many depolarizing resonances as they are accelerated to a high energy.

CP588, Physics with an Electron Polarized Light-Ion Collider, edited by R. G. Milner (c) 2001 American Institute of Physics 0-7354-0028-8/01/\$18.00 
An elegant method to overcome these depolarizing resonances, proposed by Derbenev and Kondratenko in 1976 [1], involves using a spin rotator called the Siberian snake that makes $\nu_{s}$ equal to $1 / 2$ independent of the beam energy. This energyindependent $\nu_{s}$ eliminates most of the depolarizing resonances $[2,3]$.

Once a polarized proton beam is accelerated to a high energy and stored, it is important to be able to frequently reverse its polarization direction to reduce the systematic errors in various polarized scattering asymmetry experiments. Studies at the IUCF Cooler Ring [4-6] showed that these polarization reversals, or spinflips, can be done using an rf magnet, which creates an $\mathrm{rf}$ depolarizing resonance at frequency:

$$
f_{s}=f_{r f}+n f_{c}
$$

where $f_{r f}$ is the rf magnet's frequency, $f_{c}$ is the circulation frequency, and $n$ is an integer. After crossing such a resonance by varying the rf magnet's frequency from a value below the resonance to a value above the resonance, the final beam polarization $P_{f}$ is related to the initial beam polarization $P_{i}$ via the Froissart-Stora formula [7]:

$$
P_{f}=P_{i}\left[2 e^{-\frac{\left(\pi \epsilon f_{c}\right)^{2}}{(\Delta f / \Delta t)}}-1\right],
$$

where $\epsilon$ is the resonance strength, and $\Delta f / \Delta t$ is the resonance crossing rate, while $\Delta f$ is the frequency's range during its linear variation time $\Delta t$. If the resonance is sufficiently strong and/or the crossing rate is sufficiently slow, the final polarization is reversed with respect to the initial polarization, while its absolute value remains almost unchanged; this is called a spin flip.

\section{SNAKE DESIGN}

A full Siberian snake could be made of either solenoids, whose strength is energydependent due to the Lorenz contraction of their longitudinal $\int B \cdot d l$, or dipoles, whose transverse field integrals do not change with energy in the particles' rest frame and whose strength is thus nearly energy-independent. Both designs have their advantages and disadvantages. A properly adjusted solenoidal snake does not cause any beam distortion, while inside a dipole snake there is a large orbit excursion, especially at low energies. However, at higher energies, the longitudinal $\int B d l$ required for a full snake, becomes very large. Also, a solenoidal snake's current must be ramped as the beam is accelerated; this is difficult with a superconducting solenoid. An important benefits of a dipole snake is that it requires a nearly fixed transverse $\int B \cdot d l$, which needs essentially no adjustment while accelerating the beam from low energies. Such a snake could be made of conventional warm dipole magnets and requires no cryogenics to operate.

As was shown in the LISS snake design [8], a good choice for a compact dipole snake to work with polarized protons is a so-called helical snake, which is shown in 
Figure 1. The helical snake's main body is an 8.6-meter-long helical dipole, which produces a $180^{\circ}$ spin rotation; two pairs of small tilted regular dipoles D1 and D2 on either end of the helix restore the beam's orbit without affecting the spin direction.

a)

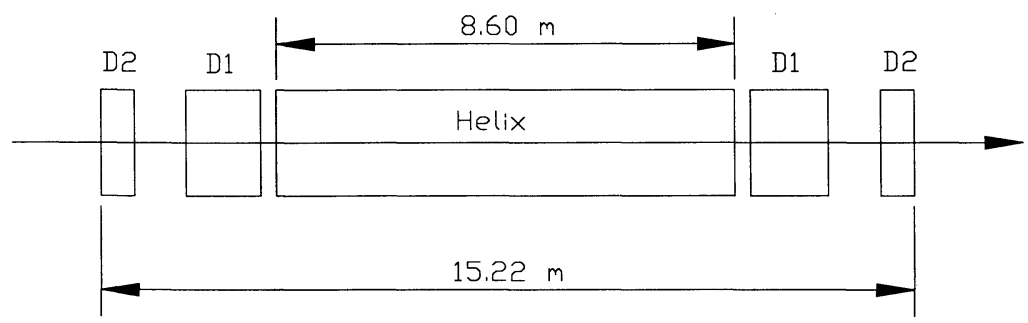

b)

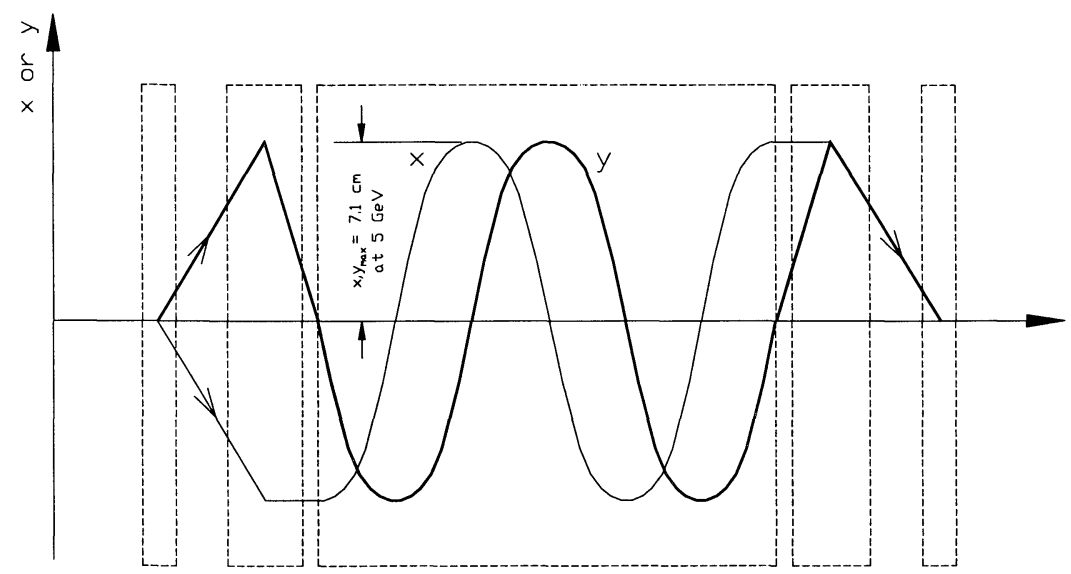

FIGURE 1. Helical Siberian snake layout (a) and beam orbit inside the snake (b).

Notice the rather small beam orbit displacement within the snake, which does not exceed $7.1 \mathrm{~cm}$ for protons at EPIC's $5 \mathrm{GeV}$ injection energy, and decreases rapidly at higher energies. This allows one to make the snake magnets' apertures quite small for lower weight and lower power consumption. A conventional SteffenLee snake, made of a series of standard dipoles, would create much larger orbit excursions, and thus needs apertures exceeding $40 \mathrm{~cm}$ [9] at EPIC's $5 \mathrm{GeV}$ injection energy. 


\section{SPIN-FLIPPER}

Of the two possible designs of an rf-magnet spin-flipper: an rf-solenoid or an rf-dipole, the rf-dipole has the very important advantage of its energy-independent spin resonance strength, while the amount of spin rotation by an rf-solenoid decreases with the particles' energy. This rf-dipole could be similar to the dipole spin-flipper, shown in Figure 2, which was designed by Michigan Spin Physics Center for the MIT-Bates South Hall electron ring. It is a ferrite-loaded single-turn magnet, whose inductance would form a tuneable parallel LC-resonant circuit with a variable capacitor (not shown). The LC-circuit would be tuned to have its resonance at the same frequency as the rf depolarizing resonance frequency in order to enhance the rf-dipole's magnetic field and thus increase its spin-flipping resonance strength. Ramping the frequency of the rf-dipole through the rf depolarizing resonance could be used to spin-flip the stored beam.

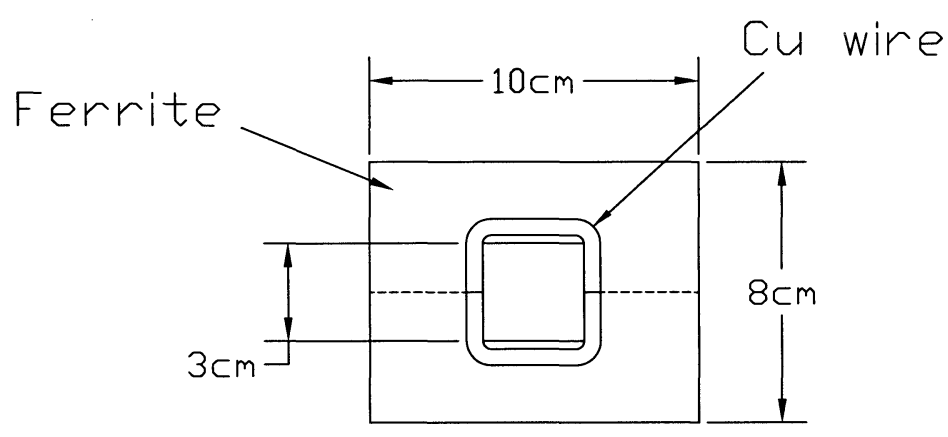

FIGURE 2. Ferrite rf-dipole spin-flipper (beam view).

However, such an rf-dipole could have a strong effect on the stored beam's betatron motion by driving coherent beam oscillations at the rf-dipole's frequency. These oscillations could be so strong that they would eventually kick the beam out of the ring's vacuum chamber aperture. Transverse beam cooling (electron cooling of the protons or synchrotron radiation cooling in the electron ring), and slow, or adiabatic, turn-on of the rf-dipole, may help reduce these oscillations and prevent the beam loss.

Another effect of such coherent beam oscillations is that they would effectively generate an additional $\mathrm{rf} \int B \cdot d l$ in the ring's quadrupole magnets, which could interfere with the rf-dipole's $\int B \cdot d l$ and could either increase the effective spindepolarizing resonance strength, or decrease it. Both effects should be taken into account in designing the actual spin-flipper. 


\section{ACKNOWLEDGMENTS}

I would like to thank A.D. Krisch and P. Schwandt for useful discussion and their help, and V.A. Anferov, Ya.S. Derbenev, T. Kageya, V.S. Morozov, D.W. Sivers, B. von Przewoski and V.K. Wong for their help. This research was supported by grants from the U.S. Department of Energy and National Science Foundation.

\section{REFERENCES}

1. Ya. S. Derbenev and A. M. Kondratenko, Sov. Phys. Dokl. 20, 562 (1976).

2. A.D. Krisch et al., Phys. Rev. Lett 63, 1137 (1989).

3. J.E. Goodwin et al., Phys. Rev. Lett. 64, 2779 (1990).

4. D.D. Caussyn et al., Phys. Rev. Lett. 73, 2857 (1994)

5. B.B Blinov et al. Phys. Rev. Lett. 81, 2906 (1998).

6. V.A. Anferov et al., Phys. Rev. ST Accel. Beams 3, 041001 (2000).

7. M. Froissart and R. Stora, Nucl. Instrum. and Methods 7, 297 (1960).

8. T.L. Gerig et al., Design of a Helical Siberian Snake for LISS, (1996).

9. V.A. Anferov et al., Acceleration of Polarized Protons to $120 \mathrm{GeV}$ and $1 \mathrm{TeV}$ at Fermilab, UM HE 95-09 (1995). 